\title{
Differences in Behavior and Brain Activity during Hypothetical and Real Choices
}

\author{
Colin Camerer ${ }^{1,2, *}$, Dean Mobbs ${ }^{1,2,3}$ \\ ${ }^{1}$ Computation and Neural Systems Program, California Institute of Technology, 1200 East \\ California Boulevard, Pasadena, CA 91125, USA \\ 2Department of Humanities and Social Sciences, California Institute of Technology, 1200 East \\ California Boulevard, Pasadena, CA 91125, USA \\ ${ }^{3}$ Department of Psychology, Columbia University, 370 Schermerhorn Hall, 1190 Amsterdam \\ Avenue, New York, NY 10027, USA
}

\begin{abstract}
Real behaviors are binding consequential commitments to a course of action, such as harming another person, buying an Apple watch, or fleeing from danger. Cognitive scientists are generally interested in the psychological and neural processes that cause such real behavior. However, for practical reasons, many scientific studies measure behavior using only hypothetical or imagined stimuli. Generalizing from such studies to real behavior implicitly assumes that the processes underlying the two types of behavior are similar. We review evidence of similarity and differences in hypothetical and real mental processes. In many cases, hypothetical choice tasks give an incomplete picture of brain circuitry that is active during real choice.
\end{abstract}

\section{Understanding How the Brain Makes Actual Choices}

Social science seeks to understand the causes of the choices that people make which affect their lives. Isolating possible causes in artificial experiments is easier when an experiment is simple, but a simple design can always be criticized on the grounds that it is too simple to be realistic. One limit on realism is that experimental subjects often make hypothetical choices with no direct consequences to them (unlike in corresponding real decisions). There may be limits to how well hypothetical choice, and associated brain activity, approximates real choice and activity. There may be similar differences between brain and behavior when objects are presented more or less realistically (e.g., a 2D image compared with an actual object).

There are likely to be two types of differences between hypothetical and real behavior: differences in naturalistic intensity of stimuli (see Glossary), and differences in what neural mechanisms are used to make choices. Consider fear as an example (discussed further later). Seeing a 2D image of a tarantula is likely to provoke less intensely arousing emotion than seeing an actual tarantula crawling toward your foot; the crawling tarantula has more

*Correspondence: camerer@ caltech.edu (C. Camerer). 
naturalistic intensity. That is, fearful emotion could be encoded in the same regions in the picture and actual conditions, but activity will be stronger and more widespread when the tarantula is real. In addition, the actual tarantula getting closer to your foot is likely to activate specialized neural circuitry (e.g., motor preparation for movement, and ancient evolutionarily conserved survival regions such as periaqueductal gray). Another example, also previewing studies discussed in the following section, is that overlapping value regions are active, whether choices are either hypothetical or real, during charitable giving and paying to avoid eating unpleasant food. However, the amygdala is only active during those choices in the real condition.

We evaluate evidence of differences in hypothetical and real behavior and brain activity in five domains: sociality, morality, emotion, economic choice, and vision. In almost all cases, there are substantial differences in behavior and brain based on the realism of stimuli (in vision) or on whether choices have actual consequences, such as financial reward, receiving shocks, buying consumer products, or eating unpleasant food.

\section{Social Neuroscience}

Social neuroscience is a relatively new field that integrates concepts from social psychology and methods from cognitive neuroscience. To date, there has been little work comparing hypothetical and real choice, but interesting work has attempted to increase the degree of realism in the form of true live interaction between multiple subjects. Social neuroscientists have studied interactions when one subject is inside an MRI scanner, and is making choices that can be influenced by interaction with one or more subjects who are outside of the scanner. Various experiments have studied empathy [1], social distance [2], social approval [3], moral behavior [4], and advice giving [5], with each pointing to new insights into the social brain. While these paradigms overcome the problems of passive observation of social stimuli (e.g., observing a face), they are not truly interactive because they reflect only the one-directional 1D outcome of one's own behavior on another person (sometimes called 'spectatorial approaches' [6] because subjects merely observe other people or contemplate the others' mental states). A 'two-directional' approach, using real-time social encounters in which all subjects make choices that influence each other's rewards, is an important step forward [7] (see Outstanding Questions).

Two-directional interactive approaches have been used in several studies. Two pioneering studies used simultaneous fMRI 'hyperscanning' of two subjects playing a financial game requiring economic trust $[7,8]$. One subject chooses how much money to invest, which triples in value; a second subject then chooses how much of that sum to share (and can keep it all) [9]. Hyperscanning was also used to study formation of 'bubbles' - price paths that grow unrealistically high and then crash. In these experimental markets, subjects chose the prices at which they trade with each other [10]. In two hyperscanning studies on bargaining, one subject first chose to say how much they would pay to buy an object, and the second subject chose whether to sell the object at that price or not [11,12]. Amygdala activity was associated with suspicion that the first subject was 'lowballing', understating what she could really pay. 
Another design [13] used live interaction between a subject being scanned and subjects outside of the scanner, while the subjects engaged in social interaction and joint attention tasks. During live social interactions, compared with recorded ones, there was more activity in many cortical mentalizing regions, including the posterior superior temporal sulcus, temporoparietal junction, and medial prefrontal cortex (mPFC). The posterior superior temporal sulcus was more active during joint compared to solo attention, supporting the idea that this region is involved in social attention.

In the 'pragmatics' approach to language, speech acts are viewed as choices intended to solve a joint action problem. Although not interactive, studies have used intersubject correlations to examine the coordination of natural speech and speech comprehension by motor, linguistic, and extralinguistic speech production systems [14,15]. In these studies, subjects are either speakers and listeners. Speakers are trained to precisely reproduce a 15min long narrative. There was a robust coupling of time-locked neural activity between speaker and listener. When speaker-listener communication failed, this neural coupling disappeared. This realistic speaker-listener coordination also found overlapping neural activity that was bilateral and more widely neurally distributed than has been observed in less lifelike tasks (which typically only show speech and comprehension overlap reliably in left hemisphere language areas). In other words, brain scanning during less realistic speech tasks gives an incomplete picture.

\section{Moral Neuroscience}

Early studies on the neural basis of moral judgment used macabre vignettes plucked from moral philosophy. An example is the 'trolley dilemma' [16]: A trolley is heading down on a track toward five people, who will be killed unless the trolley does not reach them. A subject decides whether to push a heavy man off a bridge onto the track, sacrificing one person (the heavy man) to save the five people it is barreling toward. In the contrasting scenario, the subject can throw a switch to divert the trolley from its current path to a different side track, where it will kill one person, but will save the lives of the five people on the main track. The dilemma pits a utilitarian intuition (killing one is justified by saving five) against a deontological rule (thou shalt not kill). It also varies the degree of the subject's responsibility (or so-called agency) - pushing a person compared to throwing a switch. These spectatorial paradigms have created evidence about neural regions associated with different kinds of moral judgment, under varying personal agency. However, subjects' judgments in such abstract situations could be influenced by nuisance concerns about plausibility (e.g., would a heavy body really divert a 16 000-lb trolley?) and uncontrolled filling-in of details. Decision based on self-guides, including the ideal-self (what I would ideally would choose) and ought-self (what I should or ought to choose) [17], may not forecast what people actually do in such situations [18,19].

Creating real moral dilemmas with actual consequences is constrained by ethical concerns and requires creativity, but it can be done. A motivation for using real dilemmas comes from the famous 1970s studies of obedience to [20], and abuse of [21] authority in experimental settings that were real (or subjects thought were real). Those results were surprising because 
if subjects had been asked, hypothetically, what they would do, most would predict behavior different than what was observed in the real situations.

In a recent modern study [22], the trolley dilemma was transformed to decisions about which of two groups of African orphans would have different numbers of lunches actually donated to them. Redirecting a moving ball toward one group of lunch receivers, or leaving its path toward a different group undisturbed, created the sense of personal agency corresponding to throwing the switch in the trolley problem. Activity in the insula was associated with unequal treatment of the two groups of orphans, but no strong neural correlates of intervening versus leaving the ball's path undisturbed were seen.

Another example is a so-called pain versus gain dilemma [4,23]. Subjects endowed with money could pay to reduce the level of painful shock administered to strangers (who were believed to be in an adjacent room). More money would buy a bigger reduction in the shock level. Subjects act more selfishly in the real condition - paying less money, and therefore allowing stronger shocks to others - than in an imaginary hypothetical condition. In another study, subjects underestimate how likely they are to cheat on a maths task, compared to how much they actually cheat [19]. In general, people act more like their so-called ought-self, like a moral ideal, in the hypothetical versions of these tasks (Figure 1) [24].

Brain imaging has shown a mechanistic difference between real and hypothetical moral behavior. In a hypothetical pain versus gain dilemma [4], there was increased activity in the collection of brain areas known as the imagination network, which include the posterior cingulate cortex, hippocampus, and mPFC (Box 1 and Figure 2). However, when subjects made real moral decisions, there was increased activity in the bilateral amygdala and bilateral temporoparietal junction, regions involved in negative affect and mentalizing about others. A recent study [22] compared real and hypothetical charitable donations during fMRI. They found a typical hypothetical bias - participants donate less when choices have real consequences but only in females (there is a marginally significant opposite effect in males). They found stronger amygdala activity during real choice. The insula response to viewing charity pictures before the donation decision was also predictive of whether individuals actually made donations in the real condition.

\section{Affective Neuroscience: Fear and Courage}

Most stimuli used to study human emotion are not likely to evoke all the functional human responses that are present in natural, dynamic contexts. A 2D picture of an angry face will elicit activity in threat attention circuitry (e.g., amygdala). However, in natural situations, an angry face is expressing an emotion of another person, who is in 3D and may be moving, which is likely to evoke more intense fear and preparation for flight. To observe a fuller range of neural activity in response to natural stimuli than in 2D picture studies, one group [25] had subjects view a nearby tarantula spider during fMRI. Via an artificial video feed, participants thought they were watching a live tarantula placed randomly into each of five compartments in a customized 'imminence box'. The tarantula was placed near or far away from each subject's foot. fMRI showed switches from anxiety circuits to fear circuits as the tarantula was placed closer, activity in vigilance circuits of the amygdala and bed nucleus of the stria terminalis when subjects were monitoring the movement direction of the tarantula, 
and amygdala activity in response to expectancy errors (surprise) when the tarantula was rated as scarier, during the task, than was previously predicted. These results are similar to the results of another study showing a sequence of 2D tarantula pictures every $250 \mathrm{~ms}$, which mimics the experience of a tarantula getting closer [26], although the actual 3D tarantula presence showed stronger and more distributed neural activity than the picture series.

A similar study [27] explored fear and courage, by scanning snake phobics and nonphobics while a live snake sat on a conveyer belt that could be moved nearer or farther from the back of their heads. When subjects 'courageously' made the choice to move the snake closer, their brains showed increased neural activity in the subgenual anterior cingulate cortex (ACC) and decreased activity in the amygdala. The tarantula and snake experiments go beyond simpler approaches such as showing subjects passive images of snakes and spiders, which have limited affective realism and illustrate that real threatening stimuli evoke activity in parallel circuits involved in threat assessment and overcoming one's fears.

\section{Economic Decision Making}

Real and Imagined Reward-A large number of studies in experimental psychology and economics compare hypothetical choices with real choices (almost always involving financial reward). While qualitative features of the behavior are typically close, purely hypothetical choice overstates socially desirable behavior, particularly altruism [24], cooperativeness, and patience [28] compared with real behavior under modest incentives [29].

Three neuroimaging studies explored both behavioral and circuitry differences. One study [30] compared real financial rewards in a reversal learning task with imagined rewards of a subject's own choosing (about half chose to imagine money). Real rewards activated a wide network of regions in the parietal and temporal cortices, but the conjunction of real and imagined reward activated only the medial orbitofrontal cortex. This result is evidence that the medial orbitofrontal cortex generally computes goal value, but it also implies that activation during merely imagined reward does not give the full picture of actual reward circuitry. Real rewards also exerted an undue influence in learning valuations, when equivalent hypothetical rewards also carry information about ideal choices [31]. Activity in the amygdala and ventromedial prefrontal cortex ( $\mathrm{vmPFC}$ ) is associated with a bias in favor of choosing real-rewarding stimuli, while frontal pole activity appears to suppress the bias [32]. A comparison of real and hypothetical choices of money rewards offered with shorter or longer delays (in the delay discounting paradigm) showed no differences in the rates of choosing larger delayed rewards. There were also only tiny differences in activation. Given these results, there is an obvious need for more studies comparing hypothetical and real choice in different domains.

Contingent Valuation-In economics, there is skepticism about the quality of hypothetical choice data (including many surveys) on the presumption that people will not bother to thoughtfully or accurately report what they would do unless they are motivated by incentives. This skepticism is the main reason why experimental economists always pay 
subjects extra money that depends on the choices they make [33]. A prominent way in which the difference between hypothetical and real choices influences economic practice is the 'contingent valuation method' (CVM; Box 2). CVM is a survey method for measuring the monetary values of goods and services that are not traded in markets, such as clean air or environmental damage from oil spills. However, hypothetical contingent valuations generate monetary values that seem to be very high. In a few cases, experiments have been able to actually implement choices with real consequences (e.g., buying a goose hunter's permit from them [34]) and compare those valuations with hypothetical ones for the same goods. The hypothetical monetary values are invariably higher than monetary values from real implementations (around twice as high, or more [35]).

Consumer Choice-A similar hypothetical overstatement of value is common in consumer choice: As a whole, prospective consumers typically report a probability of choosing to buy a product, which is too high compared with how often products are actually purchased [36,37]. As with CVM, many studies try to forecast actual buying rates by correcting for hypothetical bias either statistically, through instruction, or with hybrid methods [38-40]. Two studies have looked for neural differences in intensity and mechanisms between hypothetical and real purchases. Subjects either chose how much to pay for appetitive consumer products (e.g., a backpack, a wireless mouse [41]) or chose how much to pay to avoid eating aversive foods (e.g., liverwurst, 1 Tbs wasabi, [42]). In both studies, participants first make a series of hypothetical choices or valuations. Then they are surprised by a series of real decisions about other products and foods. (The decisions are real because they may actually buy a product or eat an aversive food, depending on the choices they made.) In hypothetical trials, they paid US $\$ 6.25$ more for consumer products than in real trials (from a range US\$0 to US\$50), and they paid about US\$.30 less (from a range US $\$ 0$ to US\$3) to avoid unpleasant food.

In choosing consumer goods, both hypothetical and real choices activate valuation areas in the caudate nucleus and vmPFC (in response to higher values), though brain activation is stronger and more widespread in the real choice condition. In addition, there is distinct activity in the ventral ACC only during real trials, broadly consistent with other studies showing ACC activity in conflict monitoring and behavior adjustment [43]. In valuing unpleasant foods, several areas (vmPFC, ACC, insula, amygdala, and thalamus) were significantly more activated in real decision making. Most of these regions were also correlated with disgust ratings, but only during real decision making. That is, even though the visual images of food are exactly the same, simply anticipating the prospect of actually eating the food activates distinct corticotemporal areas.

In principle, neural activity could be used to calibrate hypothetical choices to identify those which are most likely to correspond to real choices. (By analogy, single unit recording, electroencephalogram, and fMRI recording can accurately classify true memories from misrecalled ones [44].) This method could be particularly useful in predicting actual behavior when valuations are implicitly known, or likely to be self-reported with bias, such as vices and virtues (e. g., smoking and exercise) or racial discrimination [45]. Recent studies linking brain activity to later natural behavior illustrate the promise of this 'brain-as-predictor' approach to understanding natural behavior (see the section below). 
Vision

One historical way in which a possible realism gap has been described is the concept of 'ecologically valid' experimental design - that is, designs that present stimuli and decisions that resemble those in everyday life. For the study of vision, James Gibson [46] proposed the concept of ecological optics: Gibson thought that perception is not only about sensation, but is also concerned with detecting information, and that the perceiver and the environment are inseparable parts of an integrated system [46]. Modern visual neuroscience has sometimes neglected Gibson's views.

The study of the visual system has produced one of the strongest bodies of work in neuroscience. However, the visual system is embedded in a set of feed-forward and feedback networks, and is prone to misperceptions that can only be mirrored in real settings. Indeed, there is suggestive evidence that basic mechanisms change between viewing of real objects compared to pictures. (Note, by the way, that the $2 \mathrm{D}$ vs. real 3D difference is not exactly a difference between hypothetical and real, per se; it is a difference in the degree of feature realism in presentation of stimuli.) For example, when subjects repeatedly viewed real 3D objects, compared to 2D pictures of the same objects, there is differential suppression in the lateral occipital cortex, a region important in the processing of object perception. While it is still unknown what mechanisms support the differences between real and 2D processing of objects, the authors propose that real 3D objects differ from 2D pictures of objects, not only in terms of their stereoscopic depth cues, but also with respect to tangibility and the relevance of the object for grasping and interaction, which lead to differences in neural encoding [47]. Only recently did work show that visual working memory capacity, and associated duration of activation, is higher for natural objects than for artificial stimuli such as abstract color squares [48]. This type of evidence supports the Gibsonian view [46] that many studies in visual neuroscience are probing a lesser version of the real world. Little is known about how differences in visual perception relate to choice, although one behavioral study found that people valued foods and simple consumer items more when those actual objects were nearby (3D) and accessible, rather than pictured (2D) on a computer screen [49].

\section{Systematic Mistakes in Personal Choice Forecasting}

Our review so far has compared hypothetical and real choice differences in behavior, and in the brain. Another domain in which such differences could be important is in choices that have real consequences in the future. Examples include committing to do public speaking in a month, picking a college, or getting pregnant. In these cases, the initial choice does have real consequences, but it is possible that current brain activity treats the choice similar to a hypothetical one, by not mentally simulating exactly what the real future experience will be like. Indeed, social psychologists have shown errors in affective forecasting, particularly a general underestimation of the capacity of a so-called emotional immune system to cope with setbacks such as severe illness or unemployment [50,51]. In decision making, underestimating the difference between current mental states and future ones (even when they are predictably different), in a way that influences choice, is called projection bias [52]. An example of this biasis that people buy more caloric food if they shop when hungry 
[53]. Another example is charitable giving: Pledges to give for large-scale disaster relief, made when empathy is highest, are often reneged upon later (even by governments [54]). Another large body of evidence traces procrastination, and lapses of self-control to an implicit naïve hope that future decisions will be more forward-looking [55]. In these situations, even when current decisions are real, if the consequences are in the future, then neural activity may resemble hypothetical thinking - for example, by using the imagination network. To our knowledge, there is no neural evidence of these forecasting and projection biases, or whether any such biases correspond to hypothetical-real differences.

\section{Brain Activity and Natural Real-World Behavior}

Neural measures and natural behavior can be more closely linked in at least two different ways. One way is to study brain activity and structure of unusually expert subjects. Most experiments use subjects who are not expert in a task, to study decision making with no preexisting contaminating influences. However, most important real decisions are made by people who are highly expert, or at least experienced; a full account of the neural basis of choice should include measures of experts' behavior and activity. To examine the neural basis of navigation expertise, one study turned to London taxi drivers [56]. These drivers pass an examination (called The Knowledge) learning 25000 streets within a 6-mile radius of Central London. The exam is difficult: Drivers usually take an average of 34 months and 12 attempts to pass. Using voxel-based morphometry to measure gray matter density, taxi drivers had higher gray matter density in the posterior hippocampus than a control group. Density in that region also positively correlated with the length of months on the job.

A second direction, the so-called brain-as-predictor approach, correlates brain activity measured during exposure to different stimuli with subsequent actual postexperimental behavior [57-59]. For example, that to predict actual smoking 1 month later, adding activity in the $\mathrm{mPFC}$ from prediction based only on self-reported responses to viewing anti-smoking adverts doubled the $R^{2}$ from using only the self-reports to predict [58]. Other studies have predicted smoking cessation from activity in the dorsomedial prefrontal cortex [60], weight gain from response to food images [61,62], online microfinance donations from nucleus accumbens response to borrower website blurbs [63], cortisol secretion from amygdalavmPFC coupling [64], and addiction relapse. The goal is to produce increases in predictive accuracy of important natural behavior beyond self-report and other observables. The largest increases are likely to come in choice domains where correlates of behavior are revealed by brain activity, even though those predictors are implicit and inaccessible to subjects, or subjects may self-report inaccurately due to social desirability, as in addiction.

\section{Concluding Remarks}

Many areas of behavioral social science and cognitive neuroscience show differences between behavior and brain during hypothetical and real choice. The theme we have emphasized is that there are typically differences in the intensity of neural activation when subjects make real versus hypothetical choices. In addition, there are often distinct patterns of neural activation when subjects make real choices, presumably reflecting distinct neural mechanisms that are only engaged by real choices. The unpleasant implication is that studies 
based on hypothetical choice can give an incomplete picture of brain activity during real choice. For this reason, it would be valuable to have more studies comparing hypothetical and real choice within a common paradigm. Then proper meta-analysis can be done, and provide guidance about when hypothetical choice gives the most incomplete picture.

Two caveats are important to mention: First, neural activation during real and hypothetical choices is, in some cases, highly overlapping, with only small differences between these conditions (e.g., [32]). And in a nonchoice domain such as motor actions, brain scans typically show substantial overlap between activity during imagined and real movements (e. g., [65]). The simple mention of action words (e.g., kick, punch) also activates the same somatotopic motor areas as real motor actions [66]. Second, there will always be types of choices in which implementing real consequences experimentally is impossible or unethical (including highly rewarding, highly aversive, temporally distant, and morally charged choices). Experiments in these domains with hypothetical choices will obviously continue to be useful. However, any innovative methods that make stimuli more lifelike (e.g., virtual reality, or bidirectional social interactions) are likely to make evidence from even hypothetical choices a better guess about what mechanisms are involved in real choice.

\section{Acknowledgments}

Support was received from NSF and BNDF (C.C.) and NARSAD (D.M.). We thank Jody Culham and Rebecca Saxe for comments.

\section{Glossary}

\section{Affective realism}

the degree to which a laboratory stimulus evokes the same emotion it would evoke in its natural setting. For example, a picture of an angry face might capture our attention more than a neutral face, yet an angry person actually staring at us might evoke even more emotions and behaviors including extreme fear, flight or fight, and visual search for a threat source

\section{Contingent valuations}

monetary valuations that are placed on abstract goods (such as clean air, or damage from environmental disasters), which are not traded in markets. The valuations are derived 'contingent' on some hypothetical procedure, such as voting in a referendum to pay higher taxes

\section{Ecological validity}

a property of paradigms that correspond to common everyday decisions and evoke naturalistic intensity. Such paradigms capture the dynamic integration of moment-tomoment information, can be consequential, binding, and may have higher emotional intensity

\section{Naturalistic intensity}


as an experimental task or stimulus moves from artificial to naturalistic scenario, the intensity of the sensory input or subjective processing gets larger. Increased naturalistic intensity is expected to generate stronger brain activation

\section{Naturalistic-laboratory matching}

the combined use of both real natural choices and artificial laboratory choices. The experimenter creates both real world and laboratory studies and compares the results to learn how behaviors differ between the two contexts

\section{Projection bias}

the tendency for a current mental state to bias decisions with consequences in future states that are predictably different than the current state. An example is shopping while very hungry: people buy more high-calorie food they do not want to eat in the future when they are less hungry

\section{Spectatorial approach}

a term defined by Schilbach and colleagues [6] describing paradigms in which the subject is a detached observer rather than actively interacting with people or objects in the real world. Spectatorial approaches can either be unidirectional (effect of one's behaviors on others) or bidirectional (i.e., reciprocity)

\section{Two-directional interactive versus one-dimensional passive}

paradigms in which two or more subjects make decisions, which do (interactive) or do not impact other subjects (passive)

\section{References}

1. Singer T. et al. (2004) Empathy for pain involves the affective but not sensory components of pain. Science 303, 1157-1162 [PubMed: 14976305]

2. Kennedy DP et al. (2009) Personal space regulation by the human amygdala. Nat. Neurosci. 12, 1226-1227 [PubMed: 19718035]

3. Izuma K. et al. (2010) Processing of the incentive for social approval in the ventral striatum during charitable donation. J. Cogn. Neurosci. 22, 621-631 [PubMed: 19320552]

4. FeldmanHall O. et al. (2012) Differential neural circuitry and self-interest in real vs hypothetical moral decisions. Soc. Cogn. Affect. Neurosci. 7, 743-751 [PubMed: 22711879]

5. Mobbs D. et al. (2015) Reflected glory and failure: the role of the medial prefrontal cortex and ventral striatum in self vs other relevance during advice-giving outcomes. Soc. Cogn. Affect. Neurosci. 10, 1323-1328 [PubMed: 25698700]

6. Schilbach L. et al. (2013) Toward a second-person neuroscience. Behav. Brain Sci. 36, 393-414 [PubMed: 23883742]

7. King-Casas B. et al. (2008) The rupture and repair of cooperation in borderline personality disorder. Science 321, 806-810 [PubMed: 18687957]

8. King-Casas B. et al. (2005) Getting to know you: reputation and trust in a two-person economic exchange. Science 308, 78-83 [PubMed: 15802598]

9. Konvalinka I. and Roepstorff A. (2012) The two-brain approach: how can mutually interacting brains teach us something about social interaction? Front. Hum. Neurosci. 6, 215 [PubMed: 22837744]

10. Smith A. et al. (2014) Irrational exuberance and neural crash warning signals during endogenous experimental market bubbles. Proc. Natl. Acad. Sci. U.S.A. 111, 10503-10508 [PubMed: 25002476] 
11. Bhatt MA et al. (2010) Neural signatures of strategic types in a two-person bargaining game. Proc. Natl. Acad. Sci. U.S.A. 107, 19720-19725 [PubMed: 21041646]

12. Bhatt MA et al. (2012) Distinct contributions of the amygdala and parahippocampal gyrus to suspicion in a repeated bargaining game. Proc. Natl. Acad. Sci. U.S.A. 109, 8728-8733 [PubMed: 22582170]

13. Redcay E. et al. (2010) Live face-to-face interaction during fMRI: a new tool for social cognitive neuroscience. Neuroimage 50, 1639-1647 [PubMed: 20096792]

14. Silbert LJ et al. (2014) Coupled neural systems underlie the production and comprehension of naturalistic narrative speech. Proc. Natl. Acad. Sci. U.S.A. 111, E4687-E4696 [PubMed: 25267658]

15. Stephens GJ et al. (2010) Speaker-listener neural coupling underlies successful communication. Proc. Natl. Acad. Sci. U.S. A. 107, 14425-14430 [PubMed: 20660768]

16. Foot P. (1978) The Problem of Abortion and the Doctrine of the Double Effect in Virtues and Vices, Basil Blackwell

17. Higgins ET (1987) Self-discrepancy: a theory relating self and affect. Psychol. Rev. 94, 319-340 [PubMed: 3615707]

18. FeldmanHall O. and Mobbs D. (2015) A neural network for moral decision making In Brain Mapping: An Encyclopedic Reference (Toga AW, ed.), pp. 205-210, Elsevier

19. Teper R. et al. (2011) Are we more moral than we think? Exploring the role of affect in moral behavior and moral forecasting. Psychol. Sci. 22, 553-558 [PubMed: 21415242]

20. Migram S. (1974) Obedience to Authority: An Experimental View, Tavistock

21. Haney C. et al. (1973) Interpersonal dynamics in a simulated prison. Int. J. Criminol. Penology 1, 69-97

22. Hsu M. et al. (2008) The right and the good: distributive justice and neural encoding of equity and efficiency. Science 320, 1092-1095 [PubMed: 18467558]

23. FeldmanHall O. et al. (2012) What we say and what we do: the relationship between real and hypothetical moral choices. Cognition 123, 434-441 [PubMed: 22405924]

24. Engel C. (2011) Dictator games: a meta study. Exp. Econ. 14, 583-610

25. Mobbs D. et al. (2010) Neural activity associated with monitoring the oscillating threat value of a tarantula. Proc. Natl. Acad. Sci. U.S. A. 107, 20582-20586 [PubMed: 21059963]

26. Coker-Appiah DS et al. (2013) Looming animate and inanimate threats: the response of the amygdala and periaqueductal gray. Soc. Neurosci. 8, 621-630 [PubMed: 24066700]

27. Nili U. et al. (2010) Fear thou not: activity of frontal and temporal circuits in moments of real-life courage. Neuron 66, 949-962 [PubMed: 20620879]

28. Green RM and Lawyer SR (2014) Steeper delay and probability discounting of potentially real versus hypothetical cigarettes (but not money) among smokers. Behav. Processes 108, 50-56 [PubMed: 25225037]

29. Camerer CF and Hogarth RM (1999) The effects of financial incentives in experiments: a review and capital-labor-production framework. J. Risk Uncertain. 19, 7-42

30. Bray S. et al. (2010) Human medial orbitofrontal cortex is recruited during experience of imagined and real rewards. J. Neurophysiol. 103, 2506-2512 [PubMed: 20200121]

31. Scholl J. et al. (2015) The good, the bad, and the irrelevant: neural mechanisms of learning real and hypothetical rewards and effort. J. Neurosci. 35, 11233-11251 [PubMed: 26269633]

32. Bickel WK et al. (2009) Congruence of BOLD response across intertemporal choice conditions: fictive and real money gains and losses. J. Neurosci. 29, 8839-8846 [PubMed: 19587291]

33. Smith VL (1976) Experimental economics: induced value theory. Am. Econ. Rev. 66, 274-279

34. Bishop RC and Heberlein TA (1979) Measuring values of extramarket goods: are indirect measures biased? Am. J. Agric. Econ. 61, 926-930

35. Murphy JJ et al. (2005) A meta-analysis of hypothetical bias in stated preference valuation. Environ. Resour. Econ. 30, 313-325

36. Klein R. and Sherman R. (1997) Estimating new product demand from biased survey data. J. Econom. 76, 53-76 
37. Sun B. and Morwitz VG (2010) Stated intentions and purchase behavior: a unified model. Int. J. Res. Mark. 27, 356-366

38. Ding M. (2007) An incentive-aligned mechanism for conjoint analysis. J. Mark. Res. 44, 214-223

39. Ding M. et al. (2005) Incentive-aligned conjoint analysis. J. Mark. Res. 42, 67-82

40. Dong S. et al. (2010) A simple mechanism to incentive-align conjoint experiments. Int. J. Res. Mark. 27, 25-32

41. Kang MJ et al. (2011) Hypothetical and real choice differentially activate common valuation areas. J. Neurosci. 31, 461-468 [PubMed: 21228156]

42. Kang MJ and Camerer CF (2013) fMRI evidence of a hot-cold empathy gap in hypothetical and real aversive choices. Front. Neurosci. 7, 104 [PubMed: 23772205]

43. Botvinick MM (2007) Conflict monitoring and decision making: reconciling two perspectives on anterior cingulate function. Cogn. Affect. Behav. Neurosci. 7, 356-366 [PubMed: 18189009]

44. Paller KA and Wagner AD (2002) Observing the transformation of experience into memory. Trends Cogn. Sci. 6, 93-102 [PubMed: 15866193]

45. Kubota JT et al. (2012) The neuroscience of race. Nat. Neurosci. 15, 940-948 [PubMed: 22735516]

46. Gibson JJ (1979) The Ecological Approach to Visual Perception, Houghton Mifflin

47. Snow JC et al. (2011) Bringing the real world into the fMRI scanner: repetition effects for pictures versus real objects. Sci. Rep. 1, 130 [PubMed: 22355647]

48. Brady TF et al. (2016) Working memory is not fixed-capacity: more active storage capacity for real-world objects than for simple stimuli. Proc. Natl. Acad. Sci. U.S.A. 113, 7459-7464 [PubMed: 27325767]

49. Bushong B. et al. (2010) Pavlovian processes in consumer choice: the physical presence of a good increases willingnessto-pay. Am. Econ. Rev. 100, 1556-1571

50. Gilbert DT et al. (1998) Immune neglect: a source of durability bias in affective forecasting. J. Pers. Soc. Psychol. 75, 617-638 [PubMed: 9781405]

51. Miloyan B. and Suddendorf T. (2015) Feelings of the future. Trends Cogn. Sci. 19, 196-200 [PubMed: 25726365]

52. Loewenstein G. et al. (2003) Projection bias in predicting future utility. Q. J. Econ. 118, 12091248

53. Tal A. and Wansink B. (2013) Fattening fasting: hungry grocery shoppers buy more calories, not more food. JAMA Int. Med. 173, 1146-1148

54. Vidal J. and Wilson J. (2005) The Guardian 31.

55. Chabris CF et al. (2006) Intertemporal choice In The New Palgrave Dictionary of Economics (Durlauf S. and Blume L, eds), Palgrave Macmillan

56. Maguire EA et al. (2000) Navigation-related structural change in the hippocampi of taxi drivers. Proc. Natl. Acad. Sci. U.S.A. 97, 4398-4403 [PubMed: 10716738]

57. Berkman ET and Falk EB (2013) Beyond brain mapping using neural measures to predict realworld outcomes. Curr. Dir. Psychol. Sci. 22, 45-50 [PubMed: 24478540]

58. Falk EB et al. (2011) Neural activity during health messaging predicts reductions in smoking above and beyond self-report. Health Psychol. 30, 177 [PubMed: 21261410]

59. Gabrieli JD et al. (2015) Prediction as a humanitarian and pragmatic contribution from human cognitive neuroscience. Neuron 85, 11-26 [PubMed: 25569345]

60. Chua HF et al. (2011) Self-related neural response to tailored smoking-cessation messages predicts quitting. Nat. Neurosci. 14, 426 [PubMed: 21358641]

61. Demos KE et al. (2012) Individual differences in nucleus accumbens activity to food and sexual images predict weight gain and sexual behavior. J. Neurosci. 32, 5549-5552 [PubMed: 22514316]

62. Murdaugh DL et al. (2012) fMRI reactivity to high-calorie food pictures predicts short-and longterm outcome in a weight-loss program. Neuroimage 59, 2709-2721 [PubMed: 22332246]

63. Genevsky A. and Knutson B. (2015) Neural affective mechanisms predict market-level microlending. Psychol. Sci. 26, 1411-1422 [PubMed: 26187248]

Trends Cogn Sci. Author manuscript; available in PMC 2020 December 28. 
64. Urry HL et al. (2006) Amygdala and ventromedial prefrontal cortex are inversely coupled during regulation of negative affect and predict the diurnal pattern of cortisol secretion among older adults. J. Neurosci. 26, 4415-4425 [PubMed: 16624961]

65. Gerardin E. et al. (2000) Partially overlapping neural networks for real and imagined hand movements. Cereb. Cortex 10, 1093-1104 [PubMed: 11053230]

66. Hauk O. et al. (2004) Somatotopic representation of action words in human motor and premotor cortex. Neuron 41, 301-307 [PubMed: 14741110]

67. Yarkoni T. et al. (2011) Large-scale automated synthesis of human functional neuroimaging data. Nat. Methods 8, 665-670 [PubMed: 21706013]

68. Hassabis D. et al. (2007) Using imagination to understand the neural basis of episodic memory. J. Neurosci. 27, 14365-14374 [PubMed: 18160644]

69. Hassabis D. et al. (2007) Patients with hippocampal amnesia cannot imagine new experiences. Proc. Natl. Acad. Sci. U.S.A. 104, 1726-1731 [PubMed: 17229836]

70. Kwan D. et al. (2015) Cueing the personal future to reduce discounting in intertemporal choice: is episodic prospection necessary? Hippocampus 25, 432-443 [PubMed: 25676022]

71. Race E. et al. (2011) Medial temporal lobe damage causes deficits in episodic memory and episodic future thinking not attributable to deficits in narrative construction. J. Neurosci. 31, 10262-10269 [PubMed: 21753003]

72. Irish M. et al. (2012) Considering the role of semantic memory in episodic future thinking: evidence from semantic dementia. Brain 135, 2178-2191 [PubMed: 22614246]

73. Szpunar KK et al. (2014) A taxonomy of prospection: introducing an organizational framework for future-oriented cognition. Proc. Natl. Acad. Sci. U.S.A. 111, 18414-18421 [PubMed: 25416592]

74. Currie J. et al. (2015) Environmental health risks and housing values: evidence from 1,600 toxic plant openings and closings. Am. Econ. Rev. 105, 678-709 [PubMed: 27134284]

75. Kling CL et al. (2012) From exxon to BP: has some number become better than no number? J. Econ. Perspect. 26, 3-26

76. Arrow K. et al. (1993) Report of the NOAA Panel on contingent valuation. Fed. Regist. 58, 46014614

77. Loomis J. et al. (1999) Trichotomous choice: a possible solution to dual response objectives in dichotomous choice contingent valuation questions. J. Agric. Resour. Econ. 24, 572-583

78. Jacquemet N. et al. (2013) Preference elicitation under oath. J. Environ. Econ. Manage. 65, 110132

79. Hausman J. (2012) Contingent valuation: from dubious to hopeless. J. Econ. Perspect. 26, 43-56

80. Khaw MW et al. (2015) The measurement of subjective value and its relation to contingent valuation and environmental public goods. PLoS One 10, e0132842

81. Sawe N. and Knutson B. (2015) Neural valuation of environmental resources. Neuroimage 122, 87-95 [PubMed: 26265156] 
Box 1.

\section{The Imagination Network}

About 10 years ago, the idea of a distinct system for personal imagination (or 'prospection') about the future first began to gain empirical traction $[68,69]$. The motivating insight is an old one in cognitive psychology: Recalling a memory is not like opening a high-resolution JPEG file in the mind's eye; instead, a recall is a reconstruction. Since the future cannot be immediately perceived, it must be constructed, and it is plausible that memory systems are repurposed for imagining the future $[48,64,65]$.

Indeed, Hassabis et al. [68] documented an imagination network which was commonly activated by recall of both real and (previously) imagined memories, and also by newly imagined scenarios. The circuit included hippocampus bilaterally, parahippocampal gyrus, retrosplenial and posterior parietal cortices, middle temporal cortices, and medial PFC. Most of these circuit components are also evident in an original Neurosynth metaanalysis (see Figure 2 in main text). However, in [68] real memories, the precuneus, posterior cingulate cortex, and $\mathrm{mPFC}$ are more strongly activated than in imagined ones. Patients with hippocampal damage and resulting amnesia $(n=5)$ were also impaired in imagining spatially coherent scenes [69], and give less detail about future episodes than neurotypical controls [70,71].

Patients with frontotemporal dementia (FTD) typically have atrophy in the temporal pole, hippocampus, and mPFC. Since these atrophied regions are part of the imagination network, FTD patients are expected to have impairment in various kinds of future thinking; and indeed, there is mounting evidence of such impairments [72]. Notably, for this review, more ecologically valid methods that do not depend too heavily on semantic memory are needed to test for impairment in FTD patients with semantic dementia.

The next step is to understand more about overlaps and differences in the types of imagination and their behavioral function. One analysis [73] distinguished four component processes in imagination - simulation, prediction, intention, and planning and reviewed evidence about each. However, it is not known how dissociated these processes are, and how precisely they interact. Intuitively, it is likely that some of these imagination components would only be activated in real choice, so they are candidates for mechanisms that are distinct to real choice. 


\section{Box 2.}

\section{Contingent Valuation of Environmental Resources}

How much money is a pristine lake worth? While such questions may seem repugnant, such trade-offs have to be made, by governments assigning financial damages to environmental harms, and doing cost-benefit analyses (choose: condos or condors?).

Sometimes market prices can be used to infer implicit values, a method known as 'revealed preference'. For example, house prices are affected by risks from nearby plants [74]. However, when there are no market prices, surveys are used. The CVM asks people how much they value nonmarket goods in various hypothetical scenarios about resource availability or harm. CVM values are hypothetical, but are meant to match real values [75].

A 1989 US court case ruled that environmental damages could include 'nonuse' value of natural resources (e.g., valuing a park you will never visit), which can only be measured by CVM. The 1989 Exxon 'Valdez' oil spill in Alaska became a high-stakes battleground over CVM. One study, using traditional methods of estimating market-based demand for recreation, concluded that the Valdez damages were US $\$ 3.8$ million. A different study included nonuse value based on CVM surveys, and estimated damages of US $\$ 4.9$ billion [75].

The controversial Valdez case led to an influential panel of economists to establish CVM guidelines [76]. Ideal CVM studies now do variants of the following: Describe the resource decision specifically, usually multimodally (text, photos, graphics, numbers); specify a realistic market or payment mechanism; and encourage honest reporting of values [75].

Even with specific description, hypothetical CVM values are usually much larger than real values (two times or more). To correct the bias, researchers have tried statistical calibration (e.g., the National Oceanic and Atmospheric Administration proposed dividing CVMs by two [76]), or allowing expression of attitudes along with monetary value [77], or requiring a so-called solemn oath to be truthful [78]. One National Oceanic and Atmospheric Administration panelist wrote recently, 'I do not expect that proponents and opponents of contingent valuation will ever agree' (p. 54) [79].

Perhaps cognitive neuroscience can help. CVM surveys could include measures like response time, psychophysiology, facial emotion, etc. to explore biological calibration. fMRI measures of CVM valuations of environmental resources have been collected but have not yet established solid results [80,81]. 


\section{Outstanding Questions}

What are some inventive new experimental paradigms using naturalistic-laboratory matching, in which subjects interact with stimuli both in the real world and in the laboratory (to compare those behaviors)?

Can the imagination network - as mapped by prospective memory and mind wandering differentiate hypothetical and real choice activity, and map onto natural behaviors?

What methods and paradigms can establish the adaptive function of imagination and prospective memory?

Can comparisons of before and after scans of real-world training (e.g., emotion training, juggling) show how neural systems involved in hypothetical imagination are engaged and disengaged?

Can increased use of intersubject correlation methods be used to examine the properties and consequences of real-time interaction between two or more subjects (using fMRI, electroencephalogram, etc.)?

Within hypothetical choice domains, what methods can be used to distinguish trials or individuals for whom subjective experience and biological activity are more or less real?

What can be learned from expert subjects who are highly skilled in a particular task (e.g., taxi drivers, air traffic controllers, athletes) to examine how expertise circuits map onto the imagination circuit, and are linked to performance? 


\section{Trends}

Many experiments in cognitive neuroscience use hypothetical choices, or use stimuli that lack some realistic features. The goal of the experiments, however, is to understand behavior and brain activity during real choices people make.

Hypothetical and limited-realism experiments run the risk of understating the strength of brain activities, or giving an incomplete picture of the neural mechanisms, which are evoked by real choices.

There is some evidence of differences in behavior and brain activity between hypothetical and real choice in domains of social, moral, and eco- nomic choice.

There are also differences in brain activity, between more or less realistic stimuli, in emotional reactions and in visual processing.

More studies directly comparing hypothetical and real choice are needed, as well as imaginative realistic paradigms. 


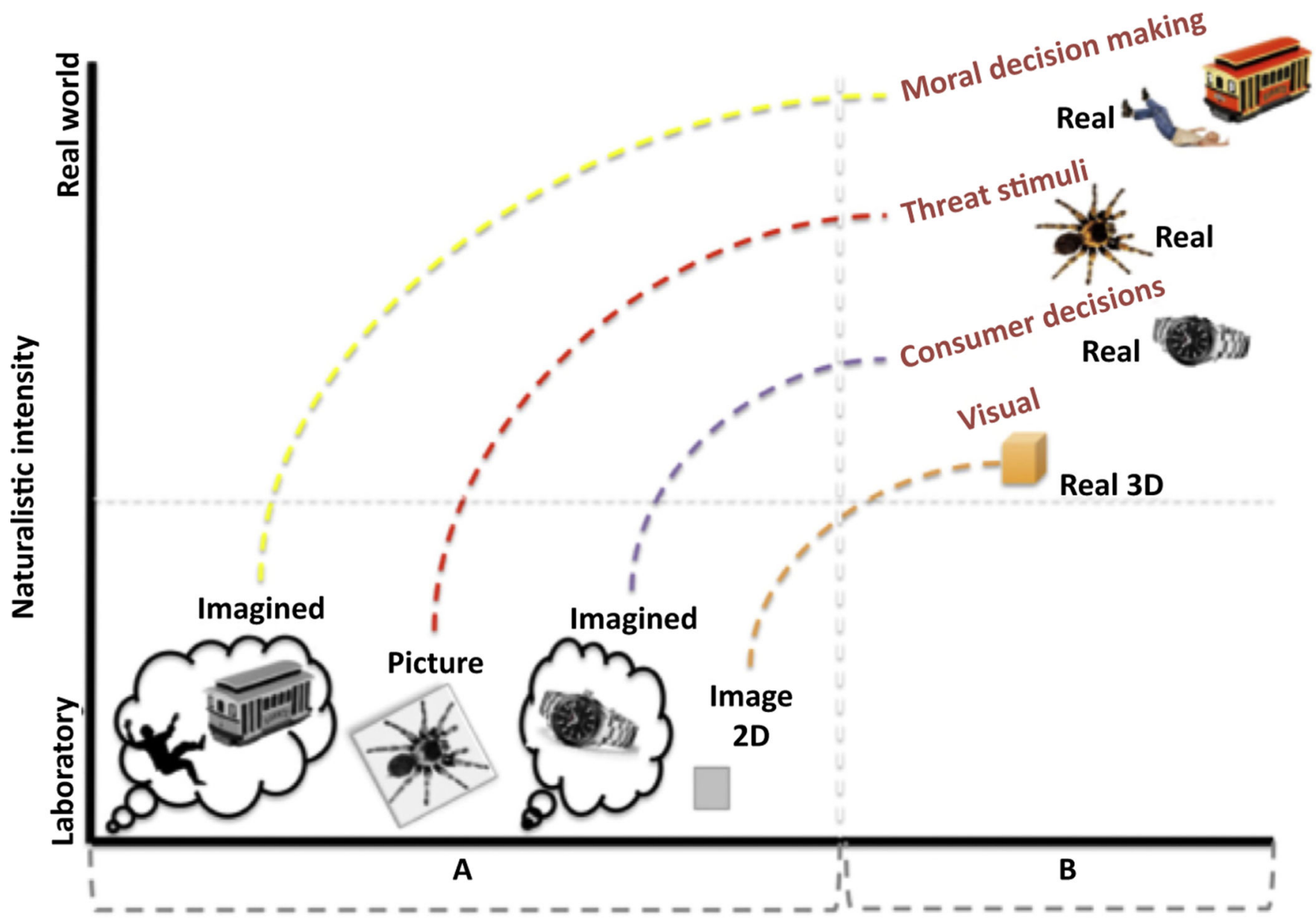

Mechanism

Trends in Cognitive Sciences

Figure 1. How Stimuli Are Hypothesized to Vary in Naturalistic Intensity and Evoked Neural Mechanisms.

The stylized graph shows naturalistic intensity on the y axis, extending from low intensity in a controlled laboratory setting to the higher intensity in natural real-world settings. Icons representing some of the studies described in this paper are all plotted low on the $y$ axis, but different laboratory paradigms could have differing degrees of naturalistic intensity. The $\mathrm{x}$ axis illustrates how some laboratory stimuli and tasks (such as real vs. hypothetical choice) will evoke different neural mechanisms. The graph illustrates the hypothesized view that many laboratory tasks are low in intensity and evoke only a subset of mechanisms, compared to more realistic choices, which are higher in intensity and evoke a larger set of mechanisms. Note that the positions of the icons and the trajectories (broken lines) do not plot actual numerical measures of intensity or mechanisms (although measures of those variables could be constructed, in principle, for some paradigms with gradation in realism). 


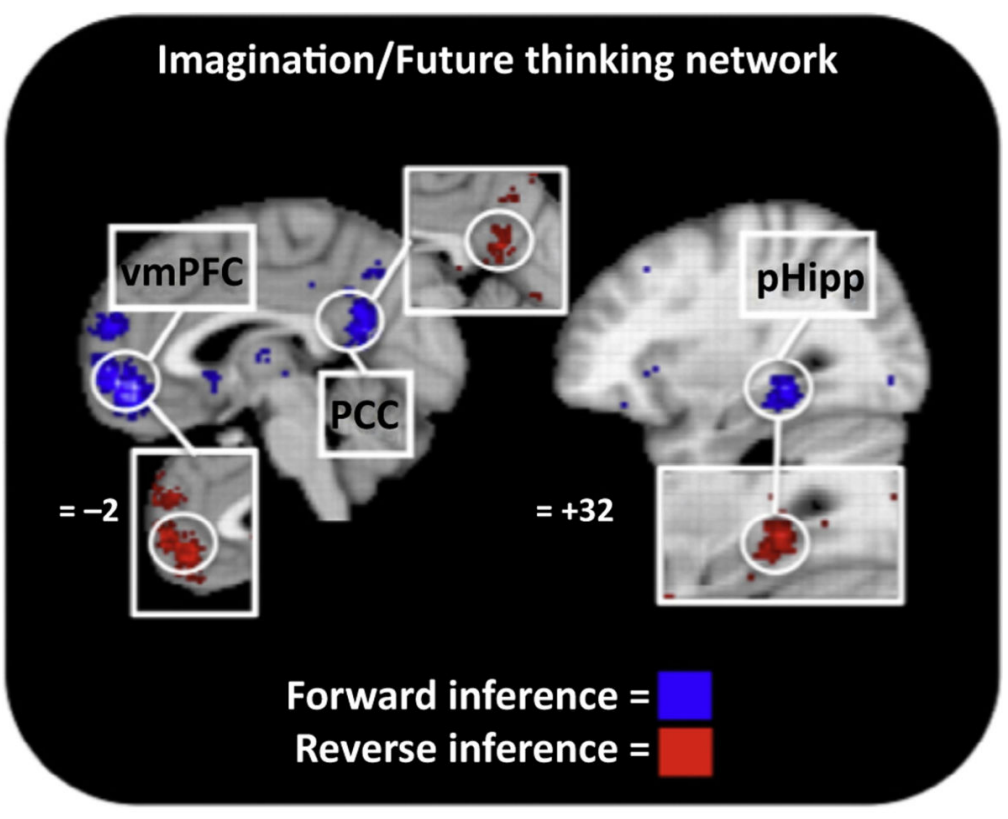

Trends in Cognitive Sciences

Figure 2. Neural Circuitry of Imagination Network.

Using Neurosynth ([67]; http://neurosynth.org), we identified 29 studies that used imagination or prospection of future events. This identified core brain regions including the posterior cingulate cortex (PCC), posterior hippocampus (pHipp), and ventromedial prefrontal cortex (mPFC). These regions have been implicated in the resting state, mind wandering, and imagination, respectively. Colored regions are those identified by forward inference (blue) or reverse inference (red). Forward inference is based on the relative frequency of studies that use a functional term (imagination), which also have activation in an area (i.e., how many studies which describe imagination find activation in the pHipp?) Reverse inference is based on the relative frequency of studies, which have an activation in a region and also use a functional term (i.e., how many studies which show activation in the pHipp say they are observing imagination?). 